\title{
The Biology of the Circadian Ckle tau Mutation in Mice and Syrian Hamsters: A Tale of Two Species
}

\author{
A.S.I. Loudon, ${ }^{*}$ Q.J. Meng, ${ }^{*}$ E.S. MaYwood, ${ }^{\dagger}$ D.A. Bechtold, ${ }^{*}$ \\ R.P. BOOT-HANDFORD, ${ }^{*}$ AND M.H. HASTINGS ${ }^{\dagger}$ \\ *Faculty of Life Sciences, University of Manchester, Manchester M13 9PT; ${ }^{\dagger}$ Division of Neurobiology, \\ MRC Laboratory of Molecular Biology, Cambridge, CB2 OQH
}

\begin{abstract}
The tau mutation in the Syrian hamster resides in the enzyme casein kinase $1 \varepsilon(\mathrm{CK} 1 \varepsilon)$, resulting in a dramatic acceleration of wheel-running activity cycles to about 20 hours. tau also impacts growth, energy, metabolism, feeding behavior, and circadian mechanisms underpinning seasonal timing, causing accelerated reproductive and neuroendocrine responses to photoperiodic changes. Modeling and experimental studies suggest that tau acts as a gain of function on specific residues of PER, consistent with hamster studies showing accelerated degradation of PER in the suprachiasmatic nucleus in the early circadian night. We have created null and tau mutants of $C k 1 \varepsilon$ in mice. Circadian period lengthens in $\mathrm{CK} 1 \varepsilon^{-/-}$, whereas $\mathrm{CK} 1 \varepsilon^{\text {tau/tau }}$ shortens circadian period of behavior in vivo in a manner nearly identical to that of the Syrian hamster. CK1 $\varepsilon^{\text {tau/tau }}$ also accelerates molecular oscillations in peripheral tissues, demonstrating its global circadian role. CK1 $\varepsilon^{\text {tau }}$ acts by promoting degradation of both nuclear and cytoplasmic PERIOD, but not CRYPTOCHROME, proteins. Our studies reveal that tau acts as a gain-of-function mutation, to accelerate degradation of PERIOD proteins. tau has consistent effects in both hamsters and mice on the circadian organization of behavior and metabolism, highlighting the global impact of this mutation on mammalian clockwork in brain and periphery.
\end{abstract}

\section{INTRODUCTION}

The past decade has witnessed spectacular advances in our understanding of the genetic basis of circadian timing in mammals. The circadian pacemakers within the suprachiasmatic nucleus ( $\mathrm{SCN}$ ) of the hypothalamus provide a crucial function in conducting a circadian repertoire throughout the body, acting on peripheral oscillators in all major body organs and tissues, with profound effects on general systemic physiology (Reppert and Weaver 2002; Hastings et al. 2003; Lowrey and Takahashi 2004; Saper et al. 2005). In both brain and periphery, the molecular clockwork operates as a series of interlocked autoregulatory feedback loops in which CLOCK:BMAL1 heterodimers bind to E-box DNA sequences contained within genes encoding transcriptional repressors PER and CRY. Following their accumulation in the cytoplasm, PER:CRY complexes translocate to the nucleus after a delay of several hours and repress the activity of constitutively bound CLOCK:BMAL1 complexes. These inhibitory complexes are then degraded following a further delay, and the consequent derepression of CLOCK:BMAL1 activity initiates the next circadian cycle of Per and Cry transcription.

We have now come to recognize the all-pervasive nature of circadian timing in biology, and the next challenge is to unravel the mechanisms and pathways involved in mediating the effects of the clocks on physiology and metabolism. The general prevalence of many diseases is also marked by a strong circadian component in morbidity, and circadian mutants are often characterized by unexpected side effects in a wide range of pathologies, including malignancy and metabolic and cardiovascular defects. Mutations in various elements of this core molecular clock have been described, many of which result in either arrhythmia or alteration in period of rest/activity and sleep cycles. Genetically mediated desynchronization of central pacemaker function is also strongly implicated as the primary causal mechanism of familial advanced sleep phase syndrome (FASPS), and unraveling the complexities of how circadian mutations act on both central and peripheral pacemakers will reveal important new insight into both the normal regulation of sleep and the pathologies associated with sleep disruption.

As in many areas of biology, much of our recent understanding has come from the use of genetically modified mice, allowing studies of incredible sophistication in a species that has an "amenable" genome. Many of the chapters in this volume are devoted to a detailed exposition of the molecular regulation of circadian timing and the impact of clocks on both normal physiology and disease processes. Here, we describe the biology of the first bona fide circadian mutation ever discovered in a mammal, the tau mutation of the Syrian hamster. We explore the impact that this timing mutation has on both the daily and seasonal biology of this species and then describe the same mutation in genetically modified mice. Here, we are able to use the power of mouse genetics to demonstrate some unexpected features of the tau gene on central timing processes in the $\mathrm{SCN}$, which reveal underpinning mechanisms controlling activity and sleep-wake cycles, with clear implications for our own species.

\section{THE DISCOVERY OF THE TAU MUTATION IN THE SYRIAN HAMSTER}

The tau mutation was discovered in 1988 by Michael Menaker and colleagues in an individual animal delivered 
as part of a routine laboratory shipment of Syrian hamsters (Mesocricetus auratus; Ralph and Menaker 1988). Subsequent breeding studies revealed that tau acts as a semidominant mutation, shortening the wheel-running period to approximately 22 hours in the heterozygote and to 20 hours in the homozygote. This spectacular and robust phenotype remains the defining feature of this iconic circadian mutant. Further studies using reciprocal SCN transplants between tau and wild-type animals provided definitive evidence that the SCN is the dominant pacemaker determining circadian wheel-running period (Ralph et al. 1990). These were extended in subsequent experiments that used microencapsulation of transplanted tissue to demonstrate that a diffusible signal of SCN origin is still capable of establishing circadian behavioral output (Silver et al. 1996). Studies of retinal melatonin rhythms also revealed an acceleration in hormone rhythms in tissue derived from tau mutants and provided crucial early evidence for the existence of additional SCN-independent oscillators (Tosini and Menaker 1996).

Following a heroic effort by Joe Takahashi and colleagues, the gene was mapped and identified as a mutation in the enzyme casein kinase $1 \varepsilon$ (CK1ع) (Lowrey et al. 2000). The team employed a comparative syntenic mapping approach, using genetically directed representational difference analysis to identify polymorphic markers that are tightly linked to a monogenic trait. This technique offered the advantage that prior knowledge of chromosomal location of the trait is unnecessary, an essential prerequisite in the unmapped mouse genome of that time. CK1 $\varepsilon$ was identified as the candidate; in mice, the gene is located on chromosome 15 and in humans, on chromosome 22. The mutation was identified as a single-base-pair C-to-T substitutional mutation, resulting in an arginine-to-cysteine transition in a highly conserved region at position 178 of CK1ع. The seven mammalian members of the CK1 family are a unique group of serine/threonine enzymes with closely conserved identity in the catalytic domain. The mutation itself occurs within the phosphate recognition domain formed by Arg-178, Gly-215, and Lys-229. The Drosophila

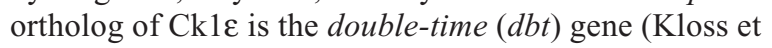
al. 1998), which has been identified in a mutagenesis screen for aberrant circadian phenotypes. Missense mutations in $d b t$ are known to confer either long or short periods on circadian behavior in flies (Price et al. 1998). In mammals, the nearest relatives of $d b t$ are two closely related members of the CK1 family: CK $1 \varepsilon$ and CK $1 \delta$. These studies thus placed CK1 as a key component in circadian timing in mammals and flies. Before exploring in more detail how CK1 $1 \varepsilon$ may accelerate circadian timing, we need first to review the impact that this mutation has upon physiology and, in particular, the endocrine and seasonal timing system of the Syrian hamster.

\section{THE IMPACT OF TAU ON NEUROENDOCRINE AND SEASONAL TIMING IN HAMSTERS}

Circadian rhythmicity is an important feature of the endocrine axis of mammals. Endocrine products of the pineal, pituitary, and adrenal glands all show marked cir- cadian cycles in synthesis and secretion, and this allows the endocrine system to impose a functionally significant temporal order on distal target tissues (Hastings et al. 2007; Maywood et al. 2007). The SCN is known to have a central role in synchronizing circadian events throughout the endocrine system, and lesions of the SCN have long been known to disrupt rhythmic hormone secretion (Moore and Eichler 1972). The accelerated circadian clock of the tau mutant Syrian hamster, together with its larger body size allowing serial blood sampling, has provided an excellent opportunity to characterize the impact of this circadian mutation on hormone secretion. Furthermore, because Syrian hamsters are seasonally breeding mammals, the tau mutation offers an ideal test bed to define the role of circadian timing in the control of the seasonal photoperiodic response.

\section{Ultradian and Daily Endocrine Rhythms}

The luteinizing hormone (LH) is under tight circadian control, and in female mammals, the time of LH surge is strongly gated by the circadian clock, with the consequence that SCN lesions severely disrupt LH secretion and ovulation (Chappell 2005; de la Iglesia and Schwartz 2006). In ovariectomized estrogen-implanted female hamsters, the gated LH surge is of normal duration and occurs daily at the same relative circadian time (CT6-8). By serial sampling during two circadian cycles in tau mutant animals, it was possible to show that the LH surge interval is accelerated such that it occurs every 20 hours in homozygote tau mutants (Lucas et al. 1999). Moreover, the surge itself is abnormal, with a significant twofold to threefold reduction in amplitude (Fig. 1f). tau also impacts high-frequency oscillators regulating episodic hormone release. For instance, in ovariectomized female hamsters, the ultradian oscillators regulating both episodic LH and cortisol secretion ( 25-35-minute oscillators) are significantly decelerated in the tau mutant (Loudon et al. 1994), a marked contrast with the acceleration of circadian cycles. It is possible that altered ultradian rhythmicity may reflect action of tau with hypothalamic GnRH neurons, because studies of GnRH cell lines reveal significant effects of clock gene mutations on endogenous episodic secretion (Chappell 2005). Our studies of hamsters reveal that although the overall circadian timing of the estrogen-induced LH surge appears to be accelerated in a manner equivalent to that of wheel-running behavior, both ultradian episodic LH and cortisol secretion and the LH surge amplitudes are highly abnormal in tau mutants. Together, these studies suggest that genes involved in circadian timing may have a key role in timing of endocrine release and secretion not only at the level of timed daily hormones, but also on noncircadian ultradian endocrine oscillator systems. It is still unclear whether abnormal neuroendocrine responses arise as a result of general pleiotropic effects of "clock" mutations or whether they reflect hitherto undefined actions of circadian timing on the endocrine axis in general.

In mammals, pineal melatonin secretion is strongly regulated by the circadian clock. Melatonin provides the brain with an internal hormonal representation of day length and is known to be the key signal involved in timing the mam- 

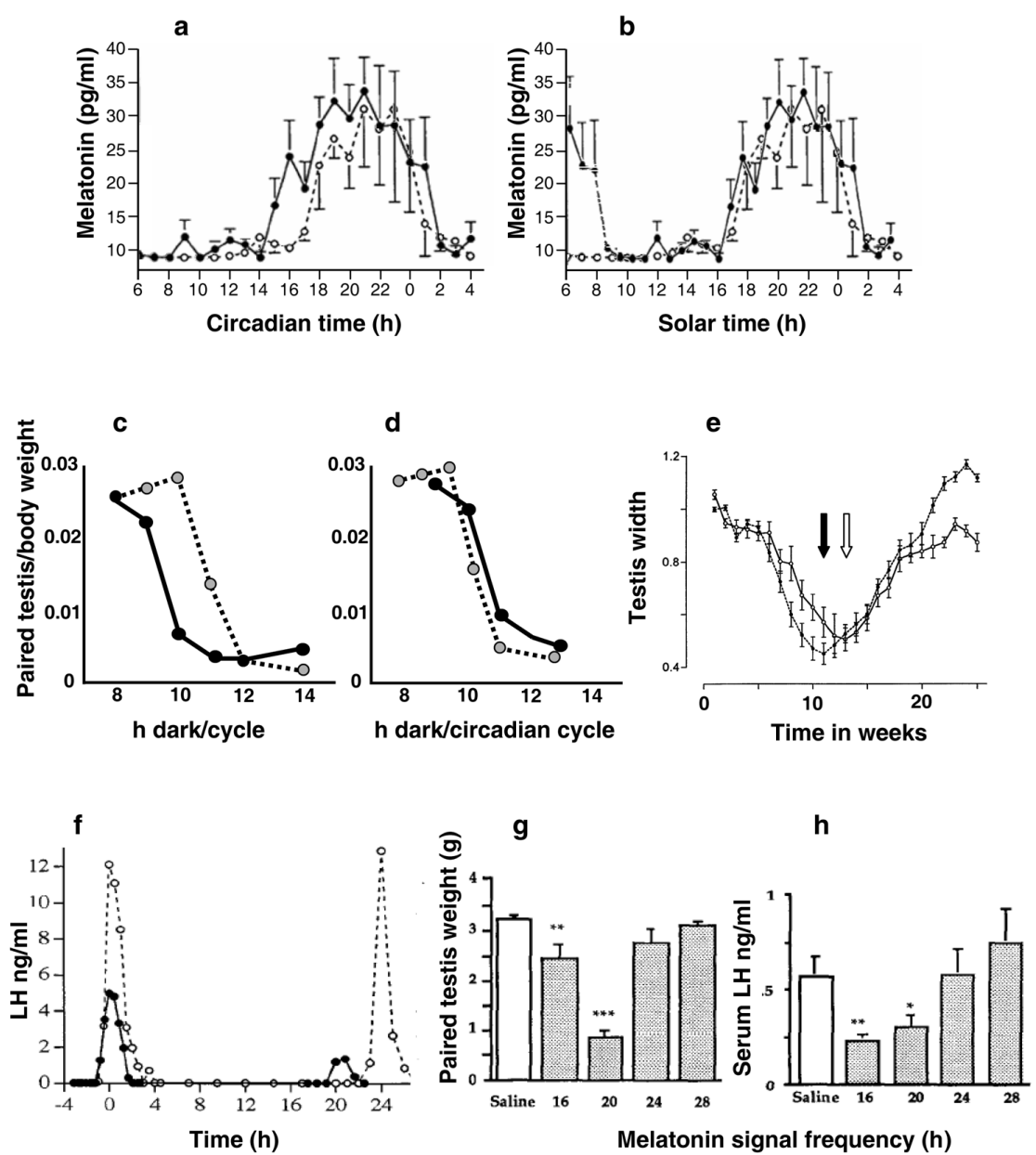

Figure 1. Seasonal and endocrine responses of tau mutant hamsters. (a) Melatonin rhythms of tau (solid line) and wild-type (dotted line) hamsters, collected every circadian hour, replotted in $(b)$ against a solar hour time base. Data are replotted in $c$ against a circadian time base. (d) Seasonal changes in testis size in tau and wild-type hamsters maintained in constant darkness (DD) for 25 weeks. (e) Paired testis weight in proportion to body size for tau mutant (solid line) and wild-type (dotted line) hamsters maintained on 20-hour (tau) or 24-hour (wild type) LD cycles. Arrows indicate the time of maximal testicular regression for tau (solid) and wild type (open arrow). $(f)$ Representative gated circadian LH surges from tau mutant (solid line) and wild-type (dotted line) ovariectomized estrogenimplanted animals sampled during two circadian cycles. $(g, h)$ Testicular and LH responses of tau mutant hamsters to a range of longduration (10 hours) melatonin signals administered at differing frequencies. (a,b,f. Redrawn, with permission, from Lucas et al. 1999; $c, e$, redrawn, with permission, from Stirland et al. 1996b; $d$, redrawn, with permission, from Loudon et al. 1998; g,h, redrawn, with permission, from Stirland et al. 1996a.)

malian neuroendocrine response to seasonal photoperiodic change: The longer nights of winter are encoded as a longer duration of the nocturnal melatonin signal (Malpaux et al. 2001). The pinealocyte itself is regulated by multisynaptic sympathetic outflow from the SCN/PVN (paraventricular nucleus), resulting in trans-synaptic release of noradrenaline and consequent regulation of $N$ acetyltransferase, the rate-limiting step in nocturnal melatonin production (Deguchi and Axelrod 1972; Axelrod 1974). In tau hamsters, the circadian onset of melatonin secretion occurs approximately 3 hours earlier (relative to activity onset) than in wild types, but curiously has a normally phased offset relative to activity onset (Lucas et al. 1999). The consequence is that despite exhibiting an accelerated circadian cycle of melatonin production (i.e., once every 20 hours), the net duration of nocturnal secretion per cycle is not significantly different in real time for each genotype. This is illustrated in Figure 1a,b where the data are plotted on both a circadian and a solar time basis. Using pineal explants, we have shown that this advance in secretion onset is not due to an altered time course of response to noradrenaline, suggesting that the timing of sympathetic activation itself and perhaps phasing of SCNmediated output may be advanced at this specific phase of the circadian cycle (Lucas et al. 1999). This has more general implications for the manner in which the tau mutation accelerates the circadian axis, which we explore in detail below in the context of the mouse model of tau.

\section{Seasonal and Photoperiodic Time Measurement}

Syrian hamsters are seasonal breeders, responsive to photoperiod and melatonin. Because the melatonin signal is generated by the circadian axis, this allows us to ask how the tau mutation might impact on photoperiodic time measurement and responsiveness to melatonin. Using tes- 
ticular regression as an index of photoresponsiveness, adult male tau mutants maintained on 24-hour light/dark (LD) cycles fail to exhibit a short-day response and undergo testicular involution, even when exposed to very short photoschedules. Instead, they exhibit a long-daylike default response (Shimomura et al. 1997). This occurs because the intrinsic 20-hour period of taus prevents their stable entrainment to the prevailing 24-hour LD cycle and thus prevents the generation of a melatonin profile representative of short days. However, when adult tau males are maintained in LD cycles on a 20-hour time base, they do entrain effectively, and when exposed to varying durations of darkness, melatonin-dependent testicular regression can be triggered by photoschedules of 10 hours light/10 hours dark. This contrasts with the threshold of 12 hours of darkness per cycle needed to trigger short-day responses in wild types and suggests that critical day-length responses are proportional to the circadian phenotype, rather than absolute (Fig. 1c,d) (Stirland et al. 1996b). One explanation of this difference is that the 10-hour night is represented by a longer melatonin profile in taus than it is in wild types due to the earlier onset of melatonin secretion $(\sim 1$ hour, rather than 4 hours after lights off). Hence, 10 hours of darkness in taus may result in the generation of the same melatonin duration as 12 hours of darkness in wild-type hamsters.

taus thus measure the passage of seasonal time using a 20 -hour time base. One consequence of this is that in 20hour lighting regimes or constant darkness (DD), melatonin signals are generated once every 20 hours (rather than every 24 hours), and correspondingly, the rate of short-day-induced physiological change is accelerated. For instance, when tau and wild-type hamsters are maintained in DD for 25 weeks, testicular regression occurs, followed by the onset of spontaneous refractoriness and recrudescence. tau mutants, however, exhibit a significantly greater rate of testicular regression, directly in proportion to the acceleration of the circadian clock (i.e., by $\sim 20 \%$ ), with the net result that they reach the nadir of the cycle 2 weeks earlier than do wild-type animals. This corresponds approximately to the time required to experience the same number of melatonin signals as lead to regression in wild type (Fig. 1e) (Loudon et al. 1998). Collectively, these data are therefore compatible with the hypothesis that acceleration in the generation of circadian melatonin signal frequency in taus leads to accelerated reproductive and neuroendocrine responses. Complementary results have been obtained in pinealectomized wild-type hamsters exposed to inhibitory long-duration melatonin signals. When these signals are administered every 20 hours, they lead to a more rapid onset of testicular regression (Maywood et al. 1990). Hence, these particular differences between tau and wild-type hamsters in photoperiodic responses to day-length and programmed melatonin signals can be explained by the impact of the mutation on circadian entrainment of the pineal melatonin profile and its photoperiodic modulation, rather than any effect of the mutation on downstream responses to melatonin.

To test whether the tau mutation has altered the definition of what constitutes a long-duration (short-night) melatonin signal or how the mutation may impair interpretation of melatonin signals of differing frequencies, we exposed pinealectomized tau and wild-type hamsters to programmed melatonin infusions every 20 hours of 8-hour duration (which are known to be inhibitory in this species) or signals of $20 \%$ shorter duration ( 6.7 hour), reduced by the same proportion by which the circadian period is shortened (Stirland et al. 1995), measuring testicular regression, serum LH, and prolactin as an end point. Both genotypes exhibited a short-day-like response, but in taus, the magnitude of response to the short-duration 6.7-hour signal was greater, with significantly reduced testis size and lowered LH levels. This implies that the mutation has altered the time base against which an inhibitory melatonin signal is measured. We have extended these studies to ask whether tau has similarly altered the time base against which melatonin signal frequencies are measured. Using the pinealectomized melatonin-infusion paradigm, we used repeated (inhibitory) long-duration (10 hours) melatonin signals administered at a range of differing signal frequencies, from 16 to 28 hours during a 6-week period (Stirland et al. 1996a). Whereas signal frequencies of 20 hours induced both significant testicular regression and suppression of LH, 16-, 24-, or 28-hour signals failed to do so. In contrast, in wild-type animals, both 20- and 24-hour infusions are effective (Fig. 1g,h). These experiments have clearly revealed that modulo-24-hour melatonin signals are not "decoded" as equivalent to short days in tau mutants, with the clear implication that the underlying ultradian interval timer that responds to the melatonin signal is itself running at an accelerated rate (see Fig. 6).

These experiments might usefully be considered in light of recent work revealing the operation of melatonininduced circadian clock gene rhythms at a melatonin neuroendocrine target site, the pituitary pars tuberalis (PT). Here, the seasonal phasing of individual components of the circadian clockwork is regulated by changes in the duration of the melatonin signal (Lincoln et al. 2002, 2003). Specifically, Cryl is strongly induced by melatonin, and onset of expression thus coincides with the early night (Hazlerigg et al. 2004), whereas the decline in melatonin at dawn leads to onset of expression of Perl (Messager et al. 1999; Lincoln et al. 2002). As a result, the melatonin-regulated clock gene rhythm in the PT target site operates as an internal coincidence timer, the interval between Cry and Per peaks being inversely proportional to day length (Lincoln et al. 2002). This has been hypothesized to lead to seasonal changes in PER/CRY-mediated repression/derepression of downstream E-box-regulated genes and hence seasonal changes in a neuroendocrine output (Lincoln et al. 2003). There is no convincing evidence that the PT operates as an autonomous circadian oscillator, rather that the molecular components of the circadian clock have been used to provide a transcriptional pathway to decode the melatonin signal. Such decoding can occur at a range of melatonin signal frequencies operating well beyond the normal range of entrainment of the SCN, because wild-type hamsters are able to read melatonin signals at 20-hour frequencies (Maywood et al. 1990). However, the alteration in response of taus to melatonin signal frequency strongly implies that changes in the kinetics of the local molecular components of the 
circadian clock at a melatonin target site may underpin failure to respond to modulo-24-hour melatonin signals. To gain insight into how the tau mutation accelerates the molecular clockwork in the Syrian hamster, we have investigated the dynamics of Perl and Per2 genes and their products during the circadian cycle.

\section{HOW DOES THE TAU MUTATION ACCELERATE CIRCADIAN TIMING? \\ PERIOD Protein Turnover in the tau Mutant Hamster}

The original paper describing the hamster mutation (Lowrey et al. 2000) proposed that tau acts as a loss of function, resulting in hypophosphorylation of target proteins. This proposition is, however, difficult to reconcile with current models of clock function, in which phosphorylation is a necessary prerequisite for targeted proteosomal degradation. One clear prediction from the biology of CK1 $1 \varepsilon$ is that the tau mutation may impact on the dynamics of clock protein turnover. To test this, we tracked both mRNA and protein for Per1 and Per2 across the circadian cycle in the SCN (Fig. 2) (Dey et al. 2005). Our data revealed that in wild-type hamsters, the rhythmic expression of PER protein exhibited a characteristic phase lag of 2-4 hours behind the mRNA cycle, with accumulation of nuclear PER proteins at the end of the subjective day and into the night. This is consistent with earlier publications in mice (Field et al. 2000). In tau mutant hamsters, there was no effect on the relative phasing of per mRNA rhythms nor on the rate or phasing of accumulation of PER proteins (counter to the predictions of accelerated accumulation based on the fly models of $d b t$ action) (Price et al. 1998). In contrast, there was an accelerated loss of immunoreactive PER, which occurs several hours earlier in the night. As a consequence of this accelerated degradation, mRNA and protein levels declined simultaneously, in contrast to wild types, which exhibit a pronounced lag in protein loss. A more detailed study taking place during the early night revealed that much of the loss of PER proteins occurred during the first 2.5 to 3 hours of the early night following the mRNA and protein peaks (Dey et al. 2005). These data are therefore consistent with a model in which CK1 $1 \varepsilon^{\text {tau }}$ leads to destabilization and degradation of nuclear PER proteins during the early subjective night, resulting in an overall acceleration of circadian period by compression of early-night phases. Such a model explains the earlier onset of melatonin secretion in tau mutant hamsters.

\section{Models for Action of $C k 1 \varepsilon$ in Circadian Timing}

In addition to periodic transcription, posttranslational modifications of clock proteins are now recognized as central components involved in the operation of molecular clockworks (Lee et al. 2001; Gallego and Virshup 2007; Mignot and Takahashi 2007). In particular, reversible phosphorylation provides a potential mechanism for the regulated formation of protein complexes, their nuclear entry, and their ultimate degradation via ubiquitination pathways, each step of which introduces delays into the feedback loop. Current interest now focuses on the role that protein phosphorylation may have in tuning the circadian oscillator to a period of 24 hours. Recent mathematical modeling studies by Forger, Virshup, and colleagues have proposed an alternative model of action to that of Lowrey et al. (2000), namely, that CK1 $\varepsilon^{\text {tau }}$ acts as a gain of function on target PER1 and PER2 proteins (Gallego et al. 2006). These authors modeled the possible actions of all known mutations of CK1 in mammals and flies to identify potential components that may contribute to period shortening. All models involving hypophosphorylation lead to lengthened, rather than shortened, period and thus
PER1-ir



PER2-ir

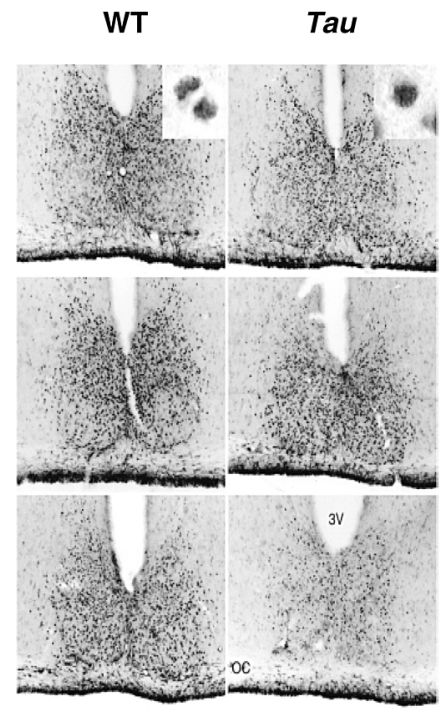

Figure 2. Immunoreactive changes in PER1 and PER2 in the SCN of the tau mutant and wild-type hamster. In tau mutants, PER proteins rapidly decline in the early nocturnal phase. (Insets) Nuclear localization for PER. (Redrawn, with permission, from Dey et al. 2005.) 
did not comply with the known biology. However, modeling of tau as a gain of function on target proteins leads to the prediction that period would shorten and that target proteins would decay at an accelerated rate. (This topic is extensively reviewed elsewhere in this volume.) The Forger/Virshup gain-of-function model was then tested experimentally in cell lines, and this revealed that $\mathrm{CK} 1 \varepsilon^{\text {tau }}$ does act as a highly specific gain-of-function mutation, which increases in vivo phosphorylation of circadian PER1 and PER2 proteins (Gallego et al. 2006). A further prediction from this model was that specific targeting of PER would lead to increased degradation, altered stability, and accelerated protein turnover, with the main effect operating on nuclear localized PER proteins (Gallego and Virshup 2007). This model is entirely consistent with our earlier data on the tau mutant hamster showing the accelerated clearance of PER at a specific phase of the cycle in tau mutants (Dey et al. 2005).

\section{The tau Mutant Mouse Model}

To test predictions for action of CK1 on core clockwork and to make use of the full power of mouse genetics, we have recently developed a mouse model for the hamster tau mutation. We adapted the loxP-Cre strategy to pro- duce mice carrying the tau allele within the $C k l \varepsilon$ gene and then used these mice subsequently to use cre-mediated in vivo disruption of exon 4 , which encodes the catalytic domain of CK1ع (Lowrey et al. 2000). This generated a frameshift resulting in a $C k l \varepsilon$ null allele (Meng et al. 2008). Wheel-running studies revealed that $\mathrm{CK} 1 \varepsilon^{\text {tau }}$ mice exhibit significant shortening of circadian period in a dose-dependent manner (see Fig. 3). This is equivalent to a reduction in circadian period of approximately 1.80 hours per copy of CK $1 \varepsilon^{\text {tau }}$, and, remarkably, this is virtually identical to the behavioral phenotype of this mutation in Syrian hamsters (Ralph and Menaker 1988). In contrast, knockout mice exhibited a small but significant period lengthening compared to their wildtype counterparts ( $\sim 18 \mathrm{~min} /$ cycle) (Fig. 3a). Importantly, tau hemizygote and heterozygote $\left(\mathrm{CK} 1 \varepsilon^{\mathrm{tau} /+}\right)$ mice exhibited similar periods (Fig. 3a,b), which reveals that a single wild-type copy of $C k l \varepsilon$ provides little protective effect in the face of a tau allele. These data are therefore consistent with the hypothesis that tau acts as a gain-of-function mutation. Furthermore, the relatively mild circadian phenotype of the knockout (slight lengthening of period, but normal intensity of activity) implies that the wild-type copy of $C k l \varepsilon$ may make relatively little contribution to normal circadian period. a

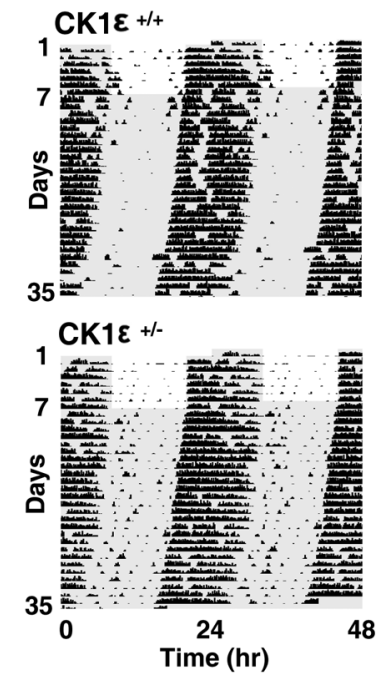

b

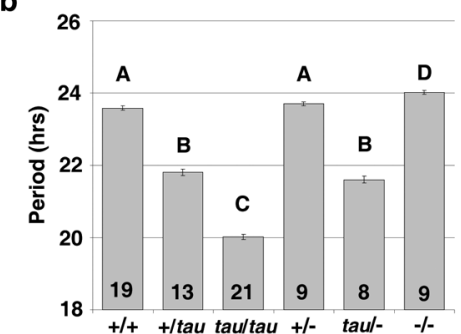

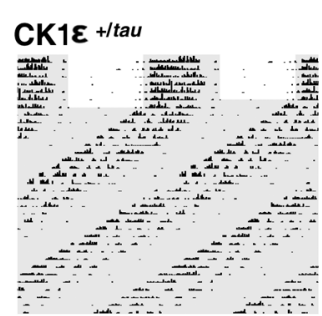

\section{CK1E taul-}

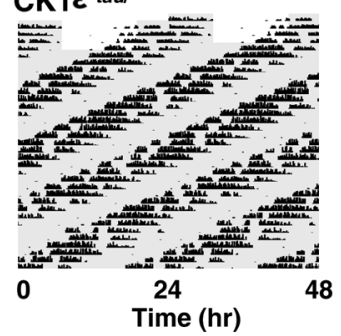

c

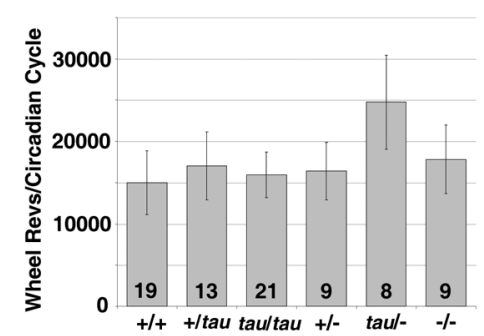

Figure 3. Locomotor activity rhythms for wild-type, tau mutant, and knockout mice. (a) Representative wheel-running activity records (actograms) for different genotypes are shown in double-plotted format. Each horizontal line represents 48 hours, with the second day plotted to the right and one cycle below the first cycle. Animals were run in 24-hour cycles of 12-hour light:12-hour dark (LD) and then in DD. The timing of the LD cycles is indicated by the alternating white and gray areas of the actogram. (b) Periodogram estimates of period for each genotype as mean \pm S.E.M.; the number of animals is indicated within each bar. Bars with different letters show a significant difference; bars with the same letter are not significantly different. (c) Activity levels (mean \pm S.E.M.) during the first 4 weeks of DD for each genotype as determined by total wheel-running revolutions per circadian cycle. (Redrawn, with permission, from Meng et al. 2008.) 
We have extended these studies by investigating the electrophysiological properties of the SCN oscillator using acutely prepared SCN slices (Meng et al. 2008). In each genotype $\left(\mathrm{CK} 1 \varepsilon^{+/+}, \mathrm{CK} 1 \varepsilon^{\text {tau/+}}, \mathrm{CK} 1 \varepsilon^{\text {tau/tau }}, \mathrm{CK} 1 \varepsilon^{-/-}\right)$, there was a close concordance with its period of multiunit and single-unit recordings in each slice (data not shown), indicating that the effects of $\mathrm{CK} 1 \varepsilon^{\text {tau }}$ on behavioral period likely arise from corresponding changes in circadian patterns of electrical activity of SCN neurons (Brown et al. 2005). Thus, the creation of the CK $1 \varepsilon^{\text {tau }}$ mutation in mice and subsequent gene deletion results in a novel allelic series of circadian periods that range from 20.0 (CK1 $\left.\varepsilon^{\text {tau/tau}}\right)$ to $24.0\left(\mathrm{CK} 1 \varepsilon^{-/-}\right)$hours and are reflected in both altered patterns of behavior and altered physiology of the SCN.

The acceleration of circadian period of activity/rest cycles as well as SCN firing rates is likely to be reflected in the altered dynamics within the molecular feedback loops that underpin circadian timekeeping within the SCN. To test this, we used a PER2::luciferase (PER2::LUC) protein fusion reporter mouse model (Yoo et al. 2004) that allows circadian monitoring of luciferase activity, accurately reporting the underlying PER2 molecular oscillator. This reporter was crossed with CK1 $1 \varepsilon^{\operatorname{tau}}$ mice, and SCN slices were recorded using bioluminescence recording (Meng et al. 2008). These studies revealed oscillations that closely matched the earlier recorded behavioral rhythms for each genotype (Fig. 4a,b): $24.4 \pm 0.15,21.9 \pm 0.08$, and $20.2 \pm 0.17$ hours in $\mathrm{CK} 1 \varepsilon^{+/+}, \mathrm{CK} 1 \varepsilon^{\text {tau } /+}$, and $\mathrm{CK} 1 \varepsilon^{\text {tau/tau }}$ slices, respectively. The overall shortening of period of the $\mathrm{SCN}$ is reflected at the single-cell level. For instance, when PER2 expression of individual SCN neurons is tracked using a CCD (charged-coupled device) camera, the resulting circadian period of single neurons also closely matches the period of the whole slice (Fig. 4c). From this, it is clear that CK $1 \varepsilon^{\text {tau }}$ has no discernible effect on the intercellular synchrony nor on the regional distribution of circadian gene expression across the SCN and is consistent with a model whereby $\mathrm{CK} 1 \varepsilon^{\text {tau }}$ accelerates all $\mathrm{SCN}$ oscillators in an equivalent manner.

\section{Action in Peripheral Tissues}

Little is known of the penetrance of many circadian mutants to peripheral tissues, and in the case of CK $1 \varepsilon^{t a u}$, the expression pattern is not known in peripheral tissues. We have tested whether endogenous CK1 $1 \varepsilon$ has a global role in setting clock speed in tissues outside the nervous system. Circadian PER2::LUC activity recording of organotypic slices of pituitary, lung, and kidney and primary lung fibroblast cultures reveals a significant shortening of period by CK1 $1 \varepsilon^{\text {tau }}$ (Fig. 4b,d). The magnitude of the effect is, however, highly variable across tissues, revealing only partial penetrance of the mutation (Meng et al. 2008). Although wild-type periods of peripheral tissues are close to 24 hours, in CK1 $1 \varepsilon^{\text {tau }}$ mice, periods are generally longer than those in the SCN by up to 2 hours, and as a result, clock speed is not accelerated to the same extent in these peripheral tissues. This suggests that mutations such as $\mathrm{CK} 1 \varepsilon^{\text {tau }}$ may induce a considerable degree of internal desynchrony in the peripheral circadian repertoire, because phased output signals driven by the SCN oscillator may act on distal oscillators of relatively longer peri- a

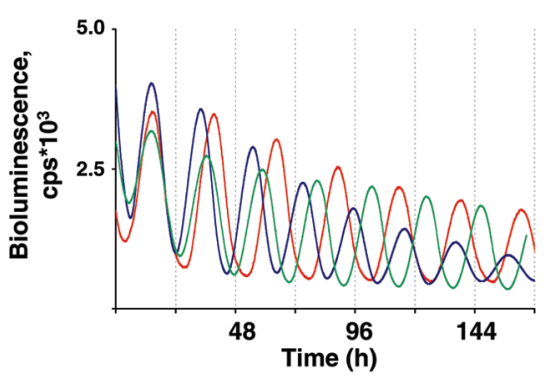

b

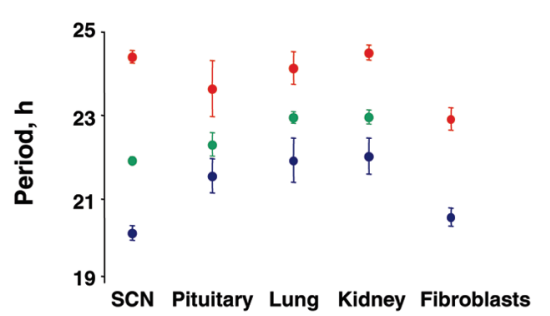

c

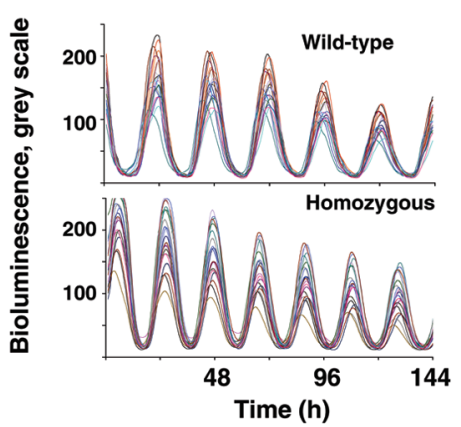

d

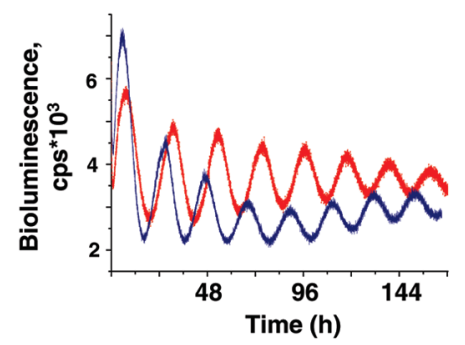

Figure 4. $\mathrm{CK} 1 \varepsilon^{t a u}$ in mice has a global impact on circadian pacemaking, accelerating molecular circadian oscillators in SCN, peripheral tissues, and primary fibroblasts. (a) Representative PER2::LUC bioluminescence oscillations in wild-type (red), heterozygote (green), and homozygote (blue) organotypic SCN slices. (b) Periodicity of tissues from wild-type (red), heterozygote (green), and homozygote (blue) tau PER2::LUC mice (mean \pm S.E.M.; wild type, $n=4-6$; heterozygote, $n=10-11$; homozygote, $n=5-6$ ). (c) CCD recordings of SCN PER2::LUC expression at the single-cell level for wild-type and homozygote SCN slices (20 per slice; $n=3$ slices for each genotype). (d) Representative traces of PER2::LUC expression in primary fibroblast cultures of wild-type (red) and tau mutant (blue) mice. (Redrawn, with permission, from Meng et al. 2008.) 
ods. One clear prediction is that peripheral clock gene expression may exhibit a different phase relationship to the central SCN oscillator. This issue is unexplored in mice, but in tau hamsters, we observed significant phase differences of Per1 and Bmall mRNA expression compared to wild type in the heart (Dey et al. 2005).

\section{Which Circadian Clock Proteins Does tau Target?}

Relatively little is known of the biochemical basis of CK1 $1 \varepsilon$ action or of the proteins targeted. To address this, we have explored the action of CK1E on PER and CRY proteins in both cell lines and primary tissues. Initially, using COS-7 cells, we used real-time fluorescence video microscopy to track PER2::YFP degradation following blockade of de novo protein synthesis by treatment with cycloheximide (CHX). Here, a clear prediction of the gain-of-function model is that coexpression with $\mathrm{CK} 1 \varepsilon^{t a u}$ kinase should accelerate PER2 degradation relative to the wild type. The data are consistent with this model (Fig. 5a), with significantly greater degradation in the face of the $\mathrm{CK} 1 \varepsilon^{\text {tau }}$ kinase. It appears that these effects are specific to PER, because CRY1::CFP degradation is not affected by any variants of CK1ع (Fig. 5b). This indicates that CK1 $\varepsilon^{\text {tau }}$ is selective for PER2. To explore whether degradation is dependent on nuclear export, we also used leptomycin B (LMB) to retain PER2::YFP in the nuclear compartment. Such treatments do not change the overall pattern of accelerated PER2 degradation by CK1 $\varepsilon^{\text {tau }}$ (Fig. $5 \mathrm{c})$ and confirm that $\mathrm{CK} 1 \varepsilon^{\text {tau }}$ acts within the nucleus. Similar dynamics appear to operate on PER1 proteins. For instance, using NIH-3T3 cells, we have retained PER1 in the nucleus or cytoplasm and examined the action of CK $1 \varepsilon^{\text {tau }}$ on degradation rates (Meng et al. 2008). Nucleartrapped PER1 was achieved by either mutation of a nuclear export signal (mtNES) or insertion of an additional copy of the nuclear localization signal (+NLS), whereas retention of PER1 in the cytoplasmic compartment was induced by use of a mutant form (PER $\left.1_{1-823}\right)$, which lacked the native nuclear localization signal, resulting in cytoplasmic retention. In all cases, CK1 $\varepsilon^{\text {tau }}$ caused accelerated degradation not only of wild-type PER1 as previously reported (Gallego et al. 2006), but also of both nuclear-trapped PER1 and cytoplasmic PER1. Together, these cell-line data clearly reveal that CK $1 \varepsilon^{\text {tau }}$ targets both PER1 and PER2 but not CRY1 proteins, and this selective targeting occurs regardless of nuclear or cytoplasmic localization.

\section{Studies of Endogenous Protein Degradation}

We have extended these studies to cells and tissue derived from mice to test whether studies of recombinant protein stability accurately reflect endogenous events. Western blots of native PER1 and CRY1 expression in wild-type and tau mutant fibroblast cultures following CHX treatment reveal that the overall protein expression of PER1 is lower in tau fibroblasts (Meng et al. 2008). Furthermore, hyperphosphorylated forms of PER1 (upper bands) display a significantly shorter half-life in tau mutant cells. These data are consistent with the concept that hyperphosphorylated PER1 may be preferentially tar- a

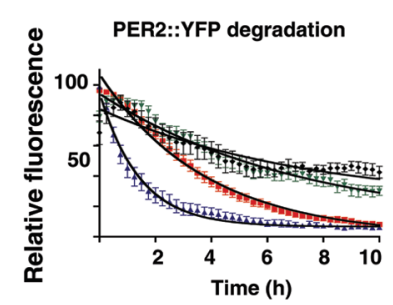

b

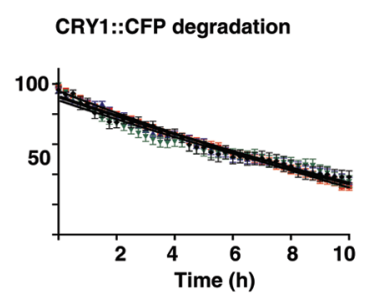

c

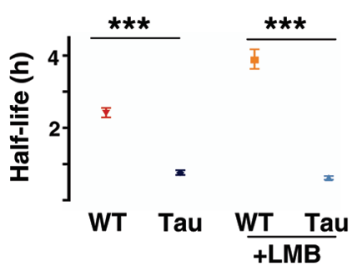

d

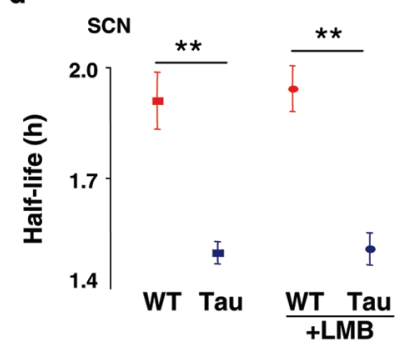

e

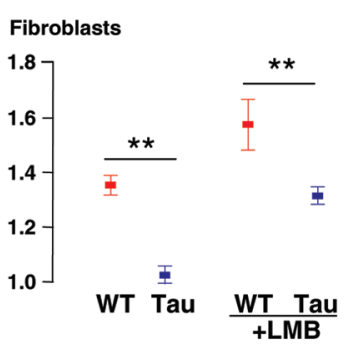

Figure 5. The tau mutation selectively targets PER proteins for accelerated degradation independently of their cellular localization. $(a, b)$ Decay of PER2::YFP or CRY1::CFP fluorescence in COS-7 cells cotransfected with wild-type (red), tau (blue), kinase-dead (green) CK1ع, or empty plasmid (black). Cells were treated with CHX $(20 \mu \mathrm{g} / \mathrm{ml}) 0.5$ hour before recording. (c) Half-lives for PER2::YFP in presence of wild-type or tau CK1 $\varepsilon$. PER2::YFP-transfected COS-7 cells were treated with vehicle or leptomycin B (LMB, $10 \mathrm{ng} / \mathrm{ml}) 1$ hour before CHX $(20 \mu \mathrm{g} / \mathrm{ml})$. (Red) CK1 $1 \varepsilon$ wild type + vehicle; (yellow) wild type + LMB; (blue) tau + vehicle; (green) tau + LMB (mean $\pm 95 \%$ confidence limits, $n=14-33$, two to four independent experiments, $* * * p<0.001$ ). (d,e) Half-lives for PER2::LUC bioluminescence from SCN slices and lung fibroblasts exposed to CHX in the presence or absence of LMB. Data are normalized to the peak level of expression (time 0 after CHX) and to the minimum after 8 hours and plotted as mean \pm S.E.M. CK1 $\varepsilon^{t a u}$ significantly accelerated loss of PER $2::$ LUC signal regardless of LMB treatment. ** $p<0.01$ by $t$-test. (Redrawn, with permission, from Meng et al. 2008.) 
geted to the proteasome for degradation (Gallego and Virshup 2007). In contrast, the overall expression level and CHX-stimulated decline of CRY1 was unaffected by $\mathrm{CK} 1 \varepsilon^{\text {tau }}$. To obtain a more dynamic view of PER degradation, bioluminescence of PER 2::LUC was recorded from SCN and lung fibroblasts treated with $\mathrm{CHX}$ at the peak of PER2 expression. This revealed that CK $1 \varepsilon^{\text {tau }}$ significantly shortened the half-life of PER2::LUC expression for both SCN and fibroblasts. Moreover, CK1 $1 \varepsilon^{\text {tau }}$ was still effective at accelerating PER2::LUC degradation in the presence of LMB (Fig. 5d,e), consistent with the recombinant protein studies that showed a nuclear action for the mutation. Together, these data provide a convincing demonstration that $\mathrm{CK} 1 \varepsilon^{\mathrm{tau}}$ facilitates the accelerated degradation of endogenous PER (but not CRY) proteins in both the SCN and primary cells and that blockade of nuclear export does not attenuate the tau phenotype.

\section{HOW DOES AN ACCELERATED CLOCK ACT ON CIRCADIAN OUTPUTS?}

Our earlier studies on the tau mutant hamster demonstrated that $\mathrm{CK} 1 \varepsilon^{\text {tau }}$ has altered the dynamics of PER protein turnover, specifically during the early-night phase of the circadian cycle. Other events (i.e., the rise of PER proteins and increase in mRNA expression) are normally phased and occur at the same solar time in both tau and wild-type animals. This early decay of the negative regulator in the SCN (PER proteins) therefore allows for early onset of nocturnal events such as melatonin secretion.
Altered dynamics of PER turnover may also offer an explanation for changes in the time base of response to repeated melatonin signals, although in the absence of a detailed understanding of how melatonin regulates these proteins at target sites, such considerations must remain speculative. One prediction from studies of PER turnover is that in the CK1 $1 \varepsilon^{\text {tau }}$ mouse, we might expect to find similar changes in the circadian dynamics of PER. We have tested this in the SCN, using the PER2::LUC reporter and aligning waveforms of SCN slices normalized to the peak or nadir of luciferase activity to identify relative changes in the rates of expression of the underlying protein. When aligned at peak expression, there is a significantly accelerated decline in signal in CK1 $1 \varepsilon^{\text {tau }}$ slices, resulting in an advance at this phase of the cycle of about 3.4 hours, compared to wild-type slices (Fig. 6) (Meng et al. 2008). Thus, much of the acceleration of period in the CK $1 \varepsilon^{\text {tau/tau }}$ mouse can be attributed to a selective early truncation of molecular events during this nocturnal phase. These data are therefore entirely consistent with our earlier studies of the tau hamster (Dey et al. 2005).

\section{Impact on Metabolism and Activity}

It appears that acceleration of molecular clockwork at a specific phase may be associated with altered circadian dynamics of behavior and physiology. For instance, in the $\mathrm{CK} 1 \varepsilon^{\text {tau }}$ mouse, the overall level of activity as reflected in total wheel-running revolutions is, somewhat remarkably, not reduced by the mutation (see Fig. 3c). This is also

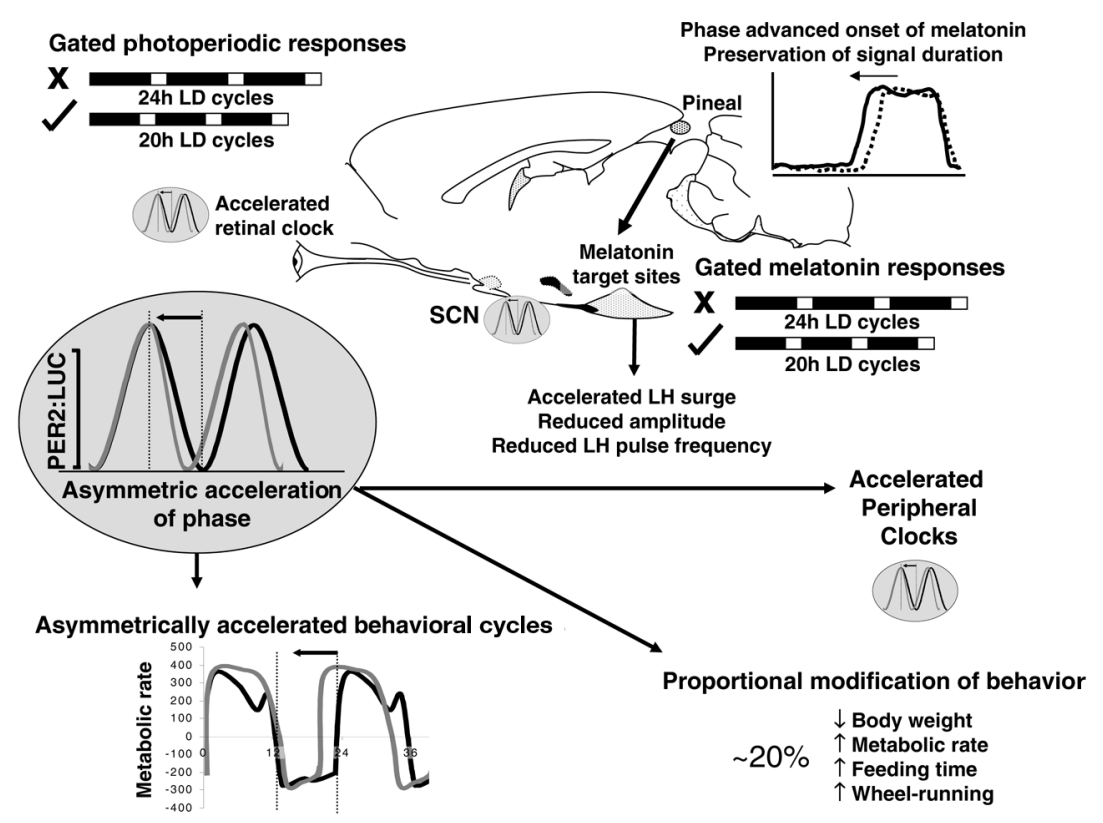

Figure 6. Summary of the impact of CK1 $1 \varepsilon^{\text {tau }}$ on circadian timing in Syrian hamsters and mice. In hamsters, the tau mutation alters the gated response to photoperiod and melatonin signals and also impacts neuroendocrine outputs (i.e., LH, cortisol). In mice, tau accelerates behavioral cycles in a manner identical to that of hamsters. In both species, this is associated with accelerated degradation of SCN PER and electrical firing rates. In both species, PER rhythms are asymmetric, with an accelerated decline in protein in the early nocturnal phase. This asymmetry results in selective compression of the diurnal phase of the cycle, such that nocturnal events (wheel-running intensity, melatonin rhythms in hamsters, and metabolic cycles in mice) are relatively unimpaired. In both species, metabolic rate is increased and body weight is reduced. Peripheral oscillators are not accelerated to the same extent as that in the SCN, leading (in hamsters) to abnormal phasing of peripheral clockwork. (PER2:LUC and metabolic rate are redrawn, with permission, from Meng et al. 2008.) 
reflected in an altered pattern of circadian energy expenditure. In $\mathrm{CK} 1 \varepsilon^{\text {tau }}$ mice, a compression of circadian rhythms of oxygen consumption $\left(\mathrm{VO}_{2}\right)$ closely matches periods determined earlier by wheel running (Meng et al. 2008). However, the pattern of oxygen consumption is highly asymmetric, such that the shortening of circadian period is almost entirely associated with a compression of the inactive (subjective day) phase (Fig. 6). The net consequence of these altered metabolic dynamics is that overall metabolic rate is increased in $\mathrm{CK} 1 \varepsilon^{\text {tau }}$ mice by approximately $20 \%$.

An identical phenotype has been reported for the tau mutant hamster. Specifically, resting metabolic rate is elevated (relative to body weight) by about $20 \%$ in homozygote tau hamsters (Oklejewicz et al. 1997), the same extent to which circadian period is accelerated, such that hamsters with only one copy of the tau allele exhibit a resting metabolic rate midway between the homozygote and wildtype animals. As a consequence, when metabolic rate is calculated per circadian cycle, no statistical differences are observed between genotypes. Oklejewicz et al. (2001) extended their studies of energy balance in tau hamsters by detailing feeding behavior and meal patterning. tau hamsters exhibited similar meal sizes, whereas total feeding time was elevated in the mutant animals. As for metabolic rate, meal frequency and total time feeding were similar between genotypes when calculated relative to the circadian cycle. Although these findings imply that both energy intake (feeding) and expenditure (metabolic rate) are proportionally increased in tau animals, altered dynamics of growth and body size are also observed in hamsters. This is not a trivial consequence of the lighting schedules in which the animals are reared, because tau hamsters grow significantly more slowly and achieve lower adult weights even when maintained, with wild-type animals as controls, in DD from birth (Lucas et al. 2000). Although we have not verified the growth rates of mutant mice during a full life cycle, adult $\mathrm{CK} 1 \varepsilon^{\mathrm{tau}}$ mice are also typically smaller than their wild-type counterparts.

A further nonintuitive but nonetheless intriguing feature of our data is that the duration of molecular night is relatively compressed due to the accelerated clearance of PER2 after its peak expression at CT12, but the duration of nocturnal behavioral and physiological processes (CBT, melatonin secretion) is apparently normal, as measured in solar time. Consequently, because the offsets are not governed by the molecular cycle, they occur several hours later than the compressed molecular cycle would predict: nocturnal processes spilling over into the start of the molecular day. Hence, the behavioral day (defined by activity offset and onset) is shorter in the mutant, even though the underlying determining change is shortening of the molecular night. These strongly asymmetric effects of $\mathrm{CK} 1 \varepsilon^{\text {tau }}$ on molecular and behavioral timing likely underlie the pronounced disturbances of circadian timekeeping (dampened amplitude, altered phasing) widely observed in the peripheral tissues of tau mutant hamsters (Dey et al. 2005) and may contribute to poor growth and general morbidity associated with the mutation in hamsters (Lucas et al. 2000). The variable responses of peripheral tissues to the tau mutation are intriguing and may suggest tissue- specific differences in the relative expression of CK1 $1 \varepsilon$ or CK1 $\delta$, or cellular differences in the manner in which PER proteins are trafficked and degraded.

Importantly, these novel phenotypes indicate that mutations in humans that alter circadian period may have complex and unpredictable effects on overall circadian organization and metabolism. This can arise either because the mutations have direct biochemical actions on metabolism or because the genetic dislocation of global circadian programs compromises the optimal functioning of particular tissues and metabolic processes, even if the mutant gene is not involved in such processes directly. Consequently, conditions such as FASPS, first recognized as sleep disorders (Toh et al. 2001; Xu et al. 2005; Vanselow et al. 2006), may in fact comprise multifactorial metabolic and physiological disturbances, echoing those recently associated with long-term rotational shift work (Knutsson 2003; Sookoian et al. 2007). This may also have implications for the development of novel pharmacological approaches to the regulation of circadian timing and phasing of sleep, because any new drugs that target these primary sleep-related complaints may also need to be tested on a wide range of circadian systems.

\section{ACKNOWLEDGMENTS}

We thank the many current and past colleagues who have worked with us over the years, including Michael Menaker, Anne Stirland, Rob Lucas, Andrei Semikhodskii, Hugh Piggins and Tim Brown, Joita Dey, Martin Sladek, Jake Lebiecki, Joe Takahashi and Seung-Hee Yoo, David Virshup and Monica Gallego, Larisa Logonova, Julia Grosse, and Jo Chesham. The work was supported by research grants from the BBSRC, MRC, and EUCLOCK.

\section{REFERENCES}

Axelrod J. 1974. The pineal gland: A neurochemical transducer. Science 184: 1341.

Brown T.M., Hughes A.T., and Piggins H.D. 2005. Gastrinreleasing peptide promotes suprachiasmatic nuclei cellular rhythmicity in the absence of vasoactive intestinal polypeptide-VPAC2 receptor signaling. J. Neurosci. 25: 11155.

Chappell P.E. 2005. Clocks and the black box: Circadian influences on gonadotropin-releasing hormone secretion. $J$. Neuroendocrinol. 17: 119.

de la Iglesia H.O. and Schwartz W.J. 2006. Minireview: Timely ovulation: Circadian regulation of the female hypothalamopituitary-gonadal axis. Endocrinology 147: 1148.

Deguchi T. and Axelrod J. 1972. Control of circadian change of serotonin $N$-acetyltransferase activity in the pineal organ by the $\beta$-adrenergic receptor. Proc. Natl. Acad. Sci. 69: 2547.

Dey J., Carr A.J., Cagampang F.R., Semikhodskii A.S., Loudon A.S., Hastings M.H., and Maywood E.S. 2005. The tau mutation in the Syrian hamster differentially reprograms the circadian clock in the SCN and peripheral tissues. J. Biol. Rhythms 20: 99.

Field M.D., Maywood E.S., O’Brien J.A., Weaver D.R., Reppert S.M., and Hastings M.H. 2000. Analysis of clock proteins in mouse SCN demonstrates phylogenetic divergence of the circadian clockwork and resetting mechanisms. Neuron 25: 437.

Gallego M. and Virshup D.M. 2007. Post-translational modifications regulate the ticking of the circadian clock. Nat. Rev. Mol. Cell Biol. 8: 139.

Gallego M., Eide E.J., Woolf M.F., Virshup D.M., and Forger D.B. 2006. An opposite role for tau in circadian rhythms 
revealed by mathematical modeling. Proc. Natl. Acad. Sci. 103: 10618.

Hastings M., O'Neill J.S., and Maywood E.S. 2007. Circadian clocks: Regulators of endocrine and metabolic rhythms. $J$. Endocrinol. 195: 187.

Hastings M.H., Reddy A.B., Garabette M., King V.M., ChahadEhlers S., O'Brien J., and Maywood E.S. 2003. Expression of clock gene products in the suprachiasmatic nucleus in relation to circadian behavior. Novartis Found. Symp. 253: 203.

Hazlerigg D.G., Andersson H., Johnston J.D., and Lincoln G. 2004. Molecular characterization of the long-day response in the Soay sheep, a seasonal mammal. Curr. Biol. 14: 334.

Kloss B., Price J.L., Saez L., Blau J., Rothenfluh A., Wesley C.S., and Young M.W. 1998. The Drosophila clock gene double-time encodes a protein closely related to human casein kinase IE. Cell 94: 97.

Knutsson A. 2003. Health disorders of shift workers. Осcup. Med. 53: 103.

Lee C., Etchegaray J.P., Cagampang F.R., Loudon A.S., and Reppert S.M. 2001. Posttranslational mechanisms regulate the mammalian circadian clock. Cell 107: 855.

Lincoln G.A., Andersson H., and Loudon A. 2003. Clock genes in calendar cells as the basis of annual timekeeping in mammals-A unifying hypothesis. J. Endocrinol. 179: 1.

Lincoln G., Messager S., Andersson H., and Hazlerigg D. 2002. Temporal expression of seven clock genes in the suprachiasmatic nucleus and the pars tuberalis of the sheep: Evidence for an internal coincidence timer. Proc. Natl. Acad. Sci. 99: 13890.

Loudon A.S., Ihara N., and Menaker M. 1998. Effects of a circadian mutation on seasonality in Syrian hamsters (Mesocricetus auratus). Proc. Biol. Sci. 265: 517.

Loudon A.S., Wayne N.L., Krieg R., Iranmanesh A., Veldhuis J.D., and Menaker M. 1994. Ultradian endocrine rhythms are altered by a circadian mutation in the Syrian hamster. Endocrinology 135: 712 .

Lowrey P.L. and Takahashi J.S. 2004. Mammalian circadian biology: Elucidating genome-wide levels of temporal organization. Annu. Rev. Genomics Hum. Genet. 5: 407.

Lowrey P.L., Shimomura K., Antoch M.P., Yamazaki S., Zemenides P.D., Ralph M.R., Menaker M., and Takahashi J.S. 2000. Positional syntenic cloning and functional characterization of the mammalian circadian mutation tau. Science 288: 483

Lucas R.J., Stirland J.A., Mohammad Y.N., and Loudon A.S. 2000. Postnatal growth rate and gonadal development in circadian tau mutant hamsters reared in constant dim red light. $J$. Reprod. Fertil. 118: 327.

Lucas R.J., Stirland J.A., Darrow J.M., Menaker M., and Loudon A.S. 1999. Free running circadian rhythms of melatonin, luteinizing hormone, and cortisol in Syrian hamsters bearing the circadian tau mutation. Endocrinology 140: 758.

Malpaux B., Migaud M., Tricoire H., and Chemineau P. 2001. Biology of mammalian photoperiodism and the critical role of the pineal gland and melatonin. J. Biol. Rhythms 16: 336 .

Maywood E.S., O’Neill J.S., Chesham J.E., and Hastings M.H. 2007. Minireview: The circadian clockwork of the suprachiasmatic nuclei-Analysis of a cellular oscillator that drives endocrine rhythms. Endocrinology 148: 5624.

Maywood E.S., Buttery R.C., Vance G.H., Herbert J., and Hastings M.H. 1990. Gonadal responses of the male Syrian hamster to programmed infusions of melatonin are sensitive to signal duration and frequency but not to signal phase nor to lesions of the suprachiasmatic nuclei. Biol. Reprod. 43: 174.

Meng Q.J., Logunova L., Maywood E.S., Gallego M., Lebiecki J., Brown T.M., Sládek M., Semikhodskii A.G., Glossop N.R.J., Piggins H.D., Chesham J.E., Bechtold D.A., Yoo S.H., Takahashi J.S., Virshup D.M., Boot-Handford R.P., Hastings M.H., and Loudon A.S.I. 2008. Setting clock speed in mammals: The CK1 $\varepsilon$ tau mutation in mice accelerates the circadian pacemaker by selectively destabilizing PERIOD proteins. Neuron (in press).
Messager S., Ross A.W., Barrett P., and Morgan P.J. 1999. Decoding photoperiodic time through Per1 and ICER gene amplitude. Proc. Natl. Acad. Sci. 96: 9938.

Mignot E. and Takahashi J.S. 2007. A circadian sleep disorder reveals a complex clock. Cell 128: 22.

Moore R.Y. and Eichler V.B. 1972. Loss of a circadian adrenal corticosterone rhythm following suprachiasmatic lesions in the rat. Brain Res. 42: 201.

Oklejewicz M., Overkamp G.J., Stirland J.A., and Daan S. 2001. Temporal organization of feeding in Syrian hamsters with a genetically altered circadian period. Chronobiol. Int. 18: 657.

Oklejewicz M., Hut R.A., Daan S., Loudon A.S., and Stirland A.J. 1997. Metabolic rate changes proportionally to circadian frequency in tau mutant Syrian hamsters. J. Biol. Rhythms. 12: 413 .

Price J.L., Blau J., Rothenfluh A., Abodeely M., Kloss B., and Young M.W. 1998. double-time is a novel Drosophila clock gene that regulates PERIOD protein accumulation. Cell 94: 83.

Ralph M.R. and Menaker M. 1988. A mutation of the circadian system in golden hamsters. Science 241: 1225.

Ralph M.R., Foster R.G., Davis F.C., and Menaker M. 1990. Transplanted suprachiasmatic nucleus determines circadian period. Science 247: 975.

Reppert S.M. and Weaver D.R. 2002. Coordination of circadian timing in mammals. Nature 418: 935.

Saper C.B., Scammell T.E., and Lu J. 2005. Hypothalamic regulation of sleep and circadian rhythms. Nature 437: 1257.

Shimomura K., Nelson D.E., Ihara N.L., and Menaker M. 1997. Photoperiodic time measurement in tau mutant hamsters. $J$. Biol. Rhythms 12: 423.

Silver R., LeSauter J., Tresco P.A., and Lehman M.N. 1996. A diffusible coupling signal from the transplanted suprachiasmatic nucleus controlling circadian locomotor rhythms. Nature 382: 810.

Sookoian S., Gemma C., Fernandez Gianotti T., Burgueno A., Alvarez A., Gonzalez C.D., and Pirola C.J. 2007. Effects of rotating shift work on biomarkers of metabolic syndrome and inflammation. J. Intern. Med. 261: 285.

Stirland J.A., Mohammad Y.N., and Loudon A.S. 1996a. A mutation of the circadian timing system (tau gene) in the seasonally breeding Syrian hamster alters the reproductive response to photoperiod change. Proc. Biol. Sci. 263: 345.

Stirland J.A., Hastings M.H., Loudon A.S., and Maywood E.S. 1996b. The tau mutation in the Syrian hamster alters the photoperiodic responsiveness of the gonadal axis to melatonin signal frequency. Endocrinology 137: 2183.

Stirland J.A., Grosse J., Loudon A.S., Hastings M.H., and Maywood E.S. 1995. Gonadal responses of the male tau mutant Syrian hamster to short-day-like programmed infusions of melatonin. Biol. Reprod. 53: 361.

Toh K.L., Jones C.R., He Y., Eide E.J., Hinz W.A., Virshup D.M., Ptáček L.J., and Fu Y.H. 2001. An hPer2 phosphorylation site mutation in familial advanced sleep phase syndrome. Science 291: 1040.

Tosini G. and Menaker M. 1996. Circadian rhythms in cultured mammalian retina. Science 272: 419.

Vanselow K., Vanselow J.T., Westermark P.O., Reischl S., Maier B., Korte T., Herrmann A., Herzel H., Schlosser A., and Kramer A. 2006. Differential effects of PER2 phosphorylation: Molecular basis for the human familial advanced sleep phase syndrome (FASPS). Genes Dev. 20: 2660.

Xu Y., Padiath Q.S., Shapiro R.E., Jones C.R., Wu S.C., Saigoh N., Saigoh K., Ptáček L.J., and Fu Y.H. 2005. Functional consequences of a CKI $\Delta$ mutation causing familial advanced sleep phase syndrome. Nature 434: 640.

Yoo S.H., Yamazaki S., Lowrey P.L., Shimomura K., Ko C.H., Buhr E.D., Siepka S.M., Hong H.K., Oh W.J., Yoo O.J., Menaker M., and Takahashi J.S. 2004. PERIOD2::LUCIFERASE real-time reporting of circadian dynamics reveals persistent circadian oscillations in mouse peripheral tissues. Proc. Natl. Acad. Sci. 101: 5339. 


\section{$\overbrace{\text { CSH' }}^{\infty}$ Cold Spring Harbor Symposia SYMPOSIA on Quantitative Biology}

\section{The Biology of the Circadian Ck1? tau Mutation in Mice and Syrian Hamsters: A Tale of Two Species}

A.S.I. Loudon, Q.J. Meng, E.S. Maywood, et al.

Cold Spring Harb Symp Quant Biol 2007 72: 261-271

Access the most recent version at doi:10.1101/sqb.2007.72.073

References This article cites 47 articles, 16 of which can be accessed free at: http://symposium.cshlp.org/content/72/261.full.html\#ref-list-1

License

Email Alerting

Receive free email alerts when new articles cite this article - sign up in the box at the Service top right corner of the article or click here.

To subscribe to Cold Spring Harbor Symposia on Quantitative Biology go to: http://symposium.cshlp.org/subscriptions 